\title{
On the extraction of notch stress intensity factors by the post-processing of stress data on the free edges of the notch
}

\author{
M Ciavarella ${ }^{1 *}$ and $\mathbf{G}$ Demelio $^{2}$ \\ ${ }^{1}$ Department of Mechanical Engineering, University of Southampton, UK \\ ${ }^{2}$ DPPI, Politecnico di Bari, Italy
}

\begin{abstract}
Following on the lines of a previous paper dedicated to cracked components by Ciavarella et al., here the case of a notch of semi-angle $\alpha$ is considered. Contrary to the crack case $\left(\alpha=180^{\circ}\right)$, the free edges of the notch are easily accessible to experimental analysis; moreover they provide information about all the terms of the Williams series expansion of the stress field about the notch apex, including the most important, i.e. the symmetric and antisymmetric singular term notch stress intensity factors (N-SIFs), whereas for the crack case the mode I N-SIFs cannot be extracted from those stresses. Another important different feature is that symmetric and antisymmetric N-SIFs have different singularities, and in several cases they are so close that their contributions tend to overlap. Therefore, a simple procedure is here proposed to use radial stresses, to separate their symmetric and antisymmetric contributions a priori by computing the sum and difference of the stresses on the two edges, to post-process these quantities in the 'asymptotic region' with standard least-squares techniques and to extract the N-SIFs. The method is applied to a simple case known in the literature and solved by means of a boundary element code, and the results are almost coincident with previous results, even with quite coarse mesh discretizations.
\end{abstract}

Keywords: sharp notches, notch stress intensity factors (N-SIFs)

\section{INTRODUCTION}

One of the classical areas of elastic theory is the wedge problem that Williams [1] studied in a celebrated paper as part of his interest in re-entrant geometries in wings and other parts of missiles. Williams considered different homogeneous boundary conditions along the radial boundaries (namely free-free, clamped-clamped and freeclamped), obtaining the classical Airy stress function as a series superposition of separate variable eigensolutions, where the radial dependence is of a power-law type (the exponents are called eigenvalues and the functions eigenvectors). Despite its widespread use in the engineering community, only recently has it been proved mathematically rigorously that the solution obtained is complete and uniformly convergent $[2,3]$. Some features of the stress field have already been discussed in reference [4], and a

The MS was received on 25 November 1998 and was accepted after revision for publication on 11 September 1999.

* Corresponding author: CNR-IRIS, Computational Mechanics of Solids (COMES), Str. Crocifisso 2/B, 70126 Bari, Italy.

S07598 (C) IMechE 2000 very detailed study of the eigenvalues has been given in reference [5].

The most 'significant' contribution to the stress field is due to the singular term which grows without limit at the notch apex [in the case of the crack $\left(\alpha=180^{\circ}\right)$, the singular terms give the well-known stress intensity factors (SIFs), and here by extension the singular terms are called notch stress intensity factors (N-SIFs)], and indeed several workers have studied techniques to extract numerically the values of these $\mathrm{N}-\mathrm{SIFs}$ [6-9]. Also, their implication in the static and fatigue strength of notched components has also been studied in recent years quite intensively [10-19]. As a crude estimate, the mode I singularity decays very slowly when the angle $\alpha$ decreases from the crack case $\left(\alpha=180^{\circ}\right)$ towards the limiting half-plane 'notch' $\left(\alpha=90^{\circ}\right)$, and therefore it is not surprising that most of the uses of SIFs for cracks extend to the notch case, particularly for those quantities that are significantly affected by the region of the notch singularity. However, two important differences are as follows:

1. As soon as a crack forms, the singularity is the wellknown square root type for the crack case $\alpha=180^{\circ}$. 
2. Mode II singularity is always lower than mode I, except for the limiting crack case $\alpha=180^{\circ}$.

Therefore, the tendency is to attribute to N-SIFs only the initiation and short crack propagation phase, and to use mostly opening criteria for these studies.

In this paper, the problem of extracting N-SIFs by an easy and, at the same time, accurate way is considered. A simple method is suggested to post-process the easily accessible radial stresses on the edges of the notch and, with a fitting procedure of the sum and difference of these stresses, the N-SIFs are obtained with good accuracy in a test case using an example case solved with boundary elements.

\section{THE STRESS FIELD}

Consider the geometry in Fig. 1. The Airy function $\Phi$ that Williams proposed for any contribution to the stress field has a separate variable form, with power-law dependence on $r$, both assumptions that can be made a priori due to self-similarity of the geometry (see Barber's book [20] for a more complete and modern treatment):

$$
\Phi=r^{\lambda+1} F(\theta ; \lambda)
$$

where

$$
\begin{aligned}
F(\theta ; \lambda)= & C_{1} \cos [(\lambda+1) \theta]+C_{2} \sin [(\lambda+1) \theta] \\
& +C_{3} \cos [(\lambda-1) \theta]+C_{4} \sin [(\lambda-1) \theta]
\end{aligned}
$$

and the stress field turns out to be

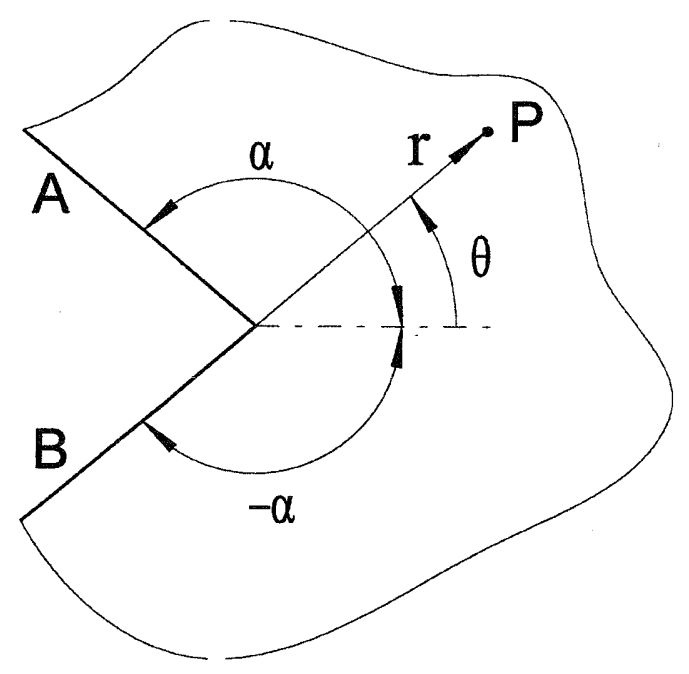

Fig. 1 Coordinate system and notation

$$
\left\{\begin{array}{c}
\sigma_{r r}(r, \theta) \\
\sigma_{\theta \theta}(r, \theta) \\
\sigma_{r \theta}(r, \theta)
\end{array}\right\}=r^{\lambda-1}\left\{\begin{array}{c}
(\lambda+1) F(\theta)+F^{\prime \prime}(\theta) \\
\lambda(\lambda+1) F(\theta) \\
-\lambda F^{\prime}(\theta)
\end{array}\right\}
$$

The coefficients $C_{i}$ and $\lambda$ are constants to be determined by imposing the boundary conditions

$$
\sigma_{r \theta}=\sigma_{\theta \theta}=0, \quad \theta= \pm \alpha
$$

obtaining a homogeneous system of four equations in the unknown $C_{i}$. As a consequence of the symmetry in the geometry and boundary conditions, symmetric and antisymmetric solutions are separated, and given by the condition that the determinant of the system should be zero. For symmetric solutions,

$$
\lambda \sin (2 \alpha)+\sin (2 \lambda \alpha)=0
$$

whereas for antisymmetric solutions

$$
\lambda \sin (2 \alpha)-\sin (2 \lambda \alpha)=0
$$

Of the entire spectrum of solutions, only those $\lambda$ giving a finite energy at the apex should be considered, i.e. $\lambda>0$.

Turning the attention to stress components, a special attention is required for complex $\lambda$, as the stress function becomes a complex function, corresponding to oscillatory functions at constant $\theta$ (both the real and the imaginary parts of the stress function are valid separately*). Summarizing, the behaviour is $[\mathbf{1}-\mathbf{5}, \mathbf{2 0}]$

$$
\sigma= \begin{cases}O\left(r^{\lambda-1}\right) & \text { real } \lambda \\
O\left(r^{\xi-1}\right)\left\{\begin{array}{l}
\sin (\eta \ln r) \\
\cos (\eta \ln r)
\end{array}\right\} & \text { complex } \lambda=\xi+\mathrm{i} \eta\end{cases}
$$

The case of complex eigenvalues is of no interest in this context as the first eigenvalue for our geometry is always real; for more complex cases such as bimaterial cracks or notches, or when the complete series expansion is required, this case is better dealt with in the context of the complex variable theory of elasticity, with the use of Mushkelishvili's potential or the Mellin transform [20], paragraphs 11.3 and 16.5)]. In the present paper, eigenvalues are computed with a standard library root finder routine [21]. At each angle, the real part of the eigenvalue is shown on the positive side of the ordinate, and the imaginary part on the negative side (Fig. 2a for symmetric and Fig. 2b for antisymmetric eigenvalues). For a given angle, moving vertically at increasing values of $\xi$, the eigenvalues are crossed in sequence. Considering firstly the symmetric terms (Fig. 2a) the first eigenvalue is real in the region of a proper notch $\left(\alpha>90^{\circ}\right)$ whereas, for non-re-entrant corners $\left(\alpha<90^{\circ}\right)$, the lowest eigenvalue is not in the singular

${ }^{*}$ For the special case at $\lambda=1$ the determinant and its derivative are zero and logarithmic terms appear, but this is not the case in wedge problems such as this. 


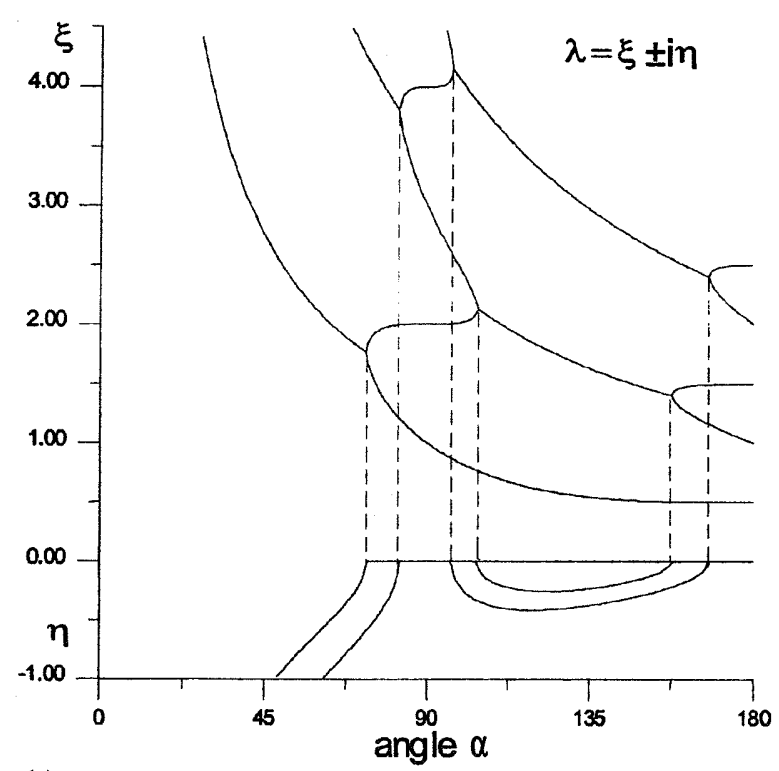

(a)

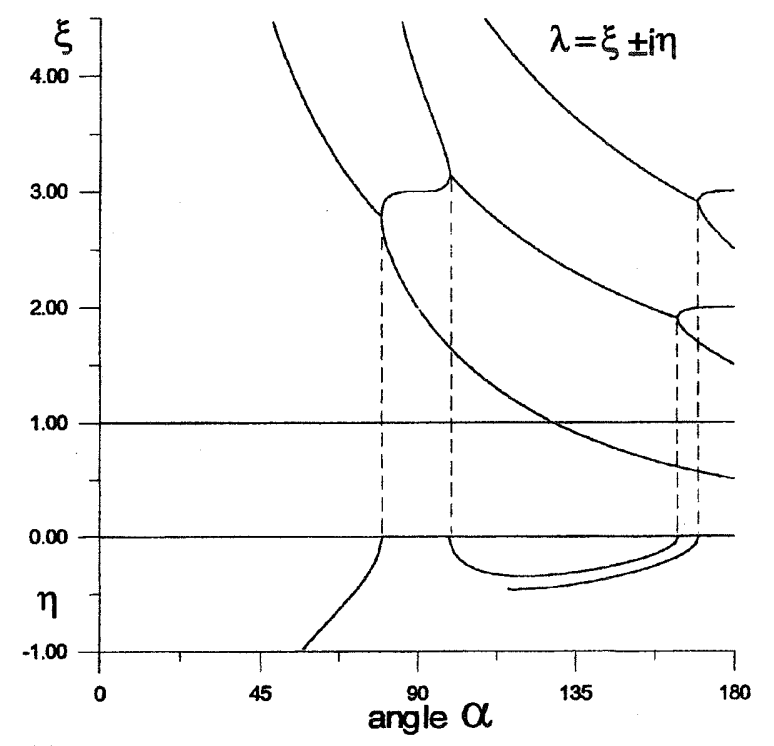

(b)

Fig. 2 Eigenvalues of the Williams expansion for (a) symmetric and (b) antisymmetric terms

region $(\xi<1)$ and becomes complex at around $\alpha=70^{\circ}$ as can be seen from the lower part of the diagram where the imaginary parts of the eigenvalues are displayed. However, the circumstance that $\xi>1$ suggests that the stress field always tends to zero at the apex in this case, and there could be little use of the complex regular term for calculation purposes. Regarding higher-order eigenvalues, the behaviour is similar to that discussed for the first eigenvalue. In particular, starting from the crack case, the eigenvalue is always real but, when the angle $\alpha$ decreases sufficiently, a region of complex eigenvalue is met when the appropriate vertical broken line is reached, after which the imaginary part of the eigenvalue can be read on the lower part of the diagram. When another broken vertical line is met, the region of complex conjugate eigenvalues ends, and another branch of real eigenvalues starts. The same qualitative behaviour obtained for antisymmetric terms is shown in Fig. $2 b$, with the significant difference that the first eigenvalue is in the singular region only for $a>127^{\circ}$, suggesting that there are notches for which only the symmetric term is present at the notch apex.

\section{POST-PROCESSING}

The stress field is given by a series of contributions according to equations (1) to (7). In particular, on the edge boundaries of the notch, radial stresses can be separated into symmetric and antisymmetric contributions. Therefore, their sum and difference will correspond to twice the symmetric part and antisymmetric part only respectively. This helps to distinguish between the contributions having close eigenvalues. From Fig. $2 \mathrm{a}$ it is evident in particular that the dominant mode I eigenvalue has almost the singularity of standard fracture mechanics if $\alpha>135^{\circ}$, i.e. $\xi=\lambda=0.5$, whereas for mode II in Fig. $2 b$ the antisymmetric term always has a quite significantly lower singularity.

$\mathrm{N}$-SIFs and higher-order terms are here defined according to reference [10], by taking advantage of the fact that on the symmetry line of the notch the circumferential stress is only due to the symmetric contribution, whereas the tangential stress is only due to the antisymmetric contribution. In particular,

$$
\sigma_{\theta \theta}(r, 0)=\sum \sigma_{\theta \theta}\left(r, 0 ; \lambda_{\mathrm{s}}\right)=\sum \frac{K_{\lambda_{\mathrm{s}}} r^{\lambda_{\mathrm{s}}-1}}{\sqrt{2 \pi}}
$$

$$
\sigma_{r \theta}(r, 0)=\sum \sigma_{r \theta}\left(r, 0 ; \lambda_{\mathrm{a}}\right)=\sum \frac{K_{\lambda_{\mathrm{a}}} r^{\lambda_{\mathrm{a}-1}}}{\sqrt{2 \pi}}
$$

where $K_{\lambda_{\mathrm{s}}}$ and $K_{\lambda_{\mathrm{a}}}$ correspond to the eigenvalues $\lambda_{\mathrm{s}}$ and $\lambda_{\mathrm{a}}$, symmetric and antisymmetric respectively.

For each symmetric contribution of the series expansion, due to the separate variable dependence of the Airy function, the ratio of the radial stress along a notch edge to the circumferential stress on the symmetry line is a constant, $R_{\lambda_{s}}$, depending only on $\alpha$ and $\lambda_{s}$. The same applies for antisymmetric functions for the ratio of the radial stress on the edge to the tangential stress on the symmetry line, $R_{\lambda_{\mathrm{s}}}$. Using equations (2) and (3), with a little algebra, 


$$
\begin{aligned}
\frac{1}{R_{\lambda_{\mathrm{s}}}} & =\frac{\sigma_{\theta \theta}\left(r, 0 ; \lambda_{\mathrm{s}}\right)}{\sigma_{r r}\left(r, \alpha ; \lambda_{\mathrm{s}}\right)} \\
& =\frac{\lambda_{\mathrm{s}}+1}{4}\left\{\frac{1}{\cos \left[\left(\lambda_{\mathrm{s}}-1\right) \alpha\right]}-\frac{1}{\cos \left[\left(\lambda_{\mathrm{s}}+1\right) \alpha\right]}\right\} \\
\frac{1}{R_{\lambda_{\mathrm{a}}}} & =\frac{\sigma_{r \theta}\left(r, 0 ; \lambda_{\mathrm{a}}\right)}{\sigma_{r r}\left(r, \alpha ; \lambda_{\mathrm{a}}\right)} \\
& =\frac{1}{4}\left\{\frac{\lambda_{\mathrm{a}}+1}{\sin \left[\left(\lambda_{\mathrm{a}}+1\right) \alpha\right]}-\frac{\lambda_{\mathrm{a}}-1}{\cos \left[\left(\lambda_{\mathrm{a}}-1\right) \alpha\right]}\right\}
\end{aligned}
$$

$R_{\lambda_{\mathrm{s}}}$ and $R_{\lambda_{\mathrm{a}}}$ for the singular eigenvalues are shown in Fig. 3 as functions of $\alpha$ varying from $90^{\circ}$ (half-plane) to $180^{\circ}$ (crack). Note that, as $R_{\lambda_{\mathrm{s}}}=0$ for the crack case, it is not possible to obtain the mode I SIF $K_{\mathrm{I}}$ from radial stresses in this case. As the vast majority of the fracture mechanics papers are dedicated to the crack case, this may well explain why this technique has not been proposed before.

In other words, it is possible now to write the radial stresses using the definitions ( $8 \mathrm{a})$ and $(8 \mathrm{~b})$ :

$$
\sigma_{r r}^{(\mathrm{s})}=\sum \frac{R_{\lambda_{\mathrm{s}}} K_{\lambda_{\mathrm{s}}} r^{\lambda_{\mathrm{s}}-1}}{\sqrt{2 \pi}}, \quad \sigma_{r r}^{(\mathrm{a})}=\sum \frac{R_{\lambda_{\mathrm{a}}} K_{\lambda_{\mathrm{a}}} r^{\lambda_{\mathrm{a}}-1}}{\sqrt{2 \pi}}
$$

where the superscripts (s) and (a) indicate the symmetric and antisymmetric contributions respectively.

\section{EXAMPLE CASE}

Consider the case in Fig. 4, for which several independent numerical results are available in the open literature [7, 9]: a rectangular plate with a deep notch of $90^{\circ}$ opening angle, under a self-equilibrated system of forces. The loads can be split into symmetric and antisymmetric contributions.

The problem is discretized with a grid of isoparametric quadratic boundary elements, with the base grid (mesh 1) indicated in Fig. 5, and more refined grids obtained by multiplying by 2,3 and 4 the number of elements on the original base grid (meshes 2, 3 and 4) and keeping the size

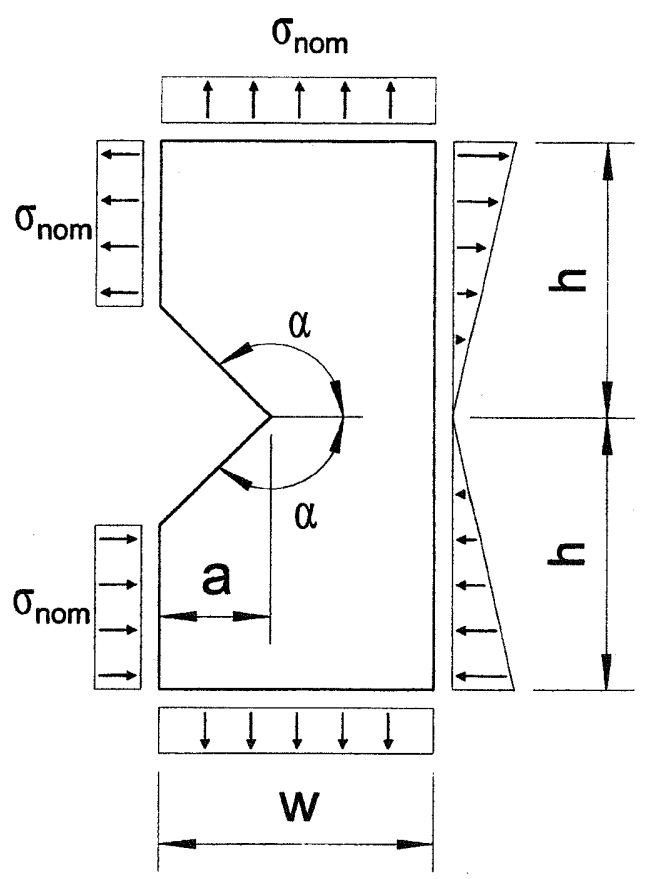

Fig. 4 Sharp notched plate loaded in the mixed mode $\left(\alpha=135^{\circ}\right.$, $w / a=2.5$ and $w / h=1$ )

ratios constant. It is clear that mesh 3 is already quite refined (with a ratio $t_{1} / t=0.02$ ). The parameters $Y$ are defined as

$$
Y_{\lambda_{\mathrm{s} 1}}=\frac{K_{\lambda_{\mathrm{s} 1}}}{\sigma_{\mathrm{nom}} \sqrt{\pi} a^{1-\lambda_{\mathrm{s} 1}}}, \quad Y_{\lambda_{\mathrm{a} 1}}=\frac{K_{\lambda_{\mathrm{al}}}}{\sigma_{\mathrm{nom}} \sqrt{\pi} a^{1-\lambda_{\mathrm{a} 1}}}
$$

To obtain these parameters from the stress boundary element method results, the following procedure is adopted. First, only the asymptotic values, which are arbitrarily decided to be in the region $r / t \in[0,0.2]$ (but this decision is not as important when later the extrapolation procedure is completed), are considered. Then, considering only the dominant term of equations (10), an estimate of the parameters $Y$ can be obtained at different distances from each stress data. Finally, using a standard extrapolation

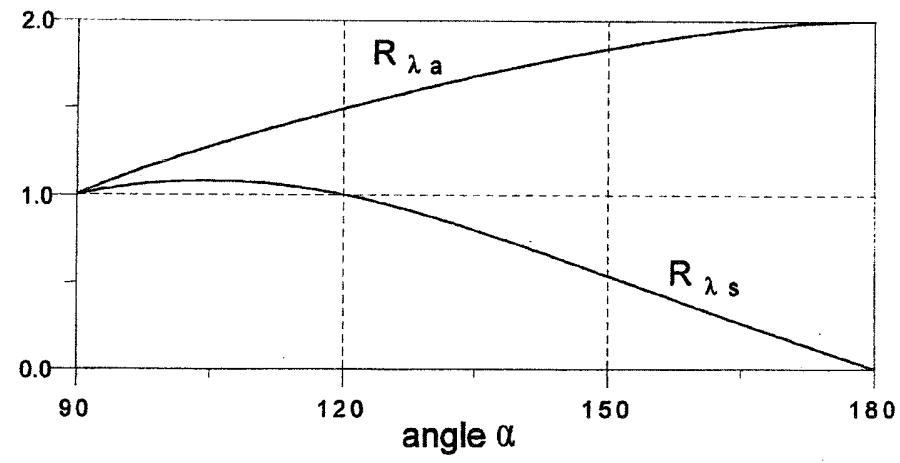

Fig. 3 Ratios $R_{\lambda_{\mathrm{s}}}$ and $R_{\lambda_{\mathrm{a}}}$ for the first symmetric and antisymmetric terms of the Williams expansion 


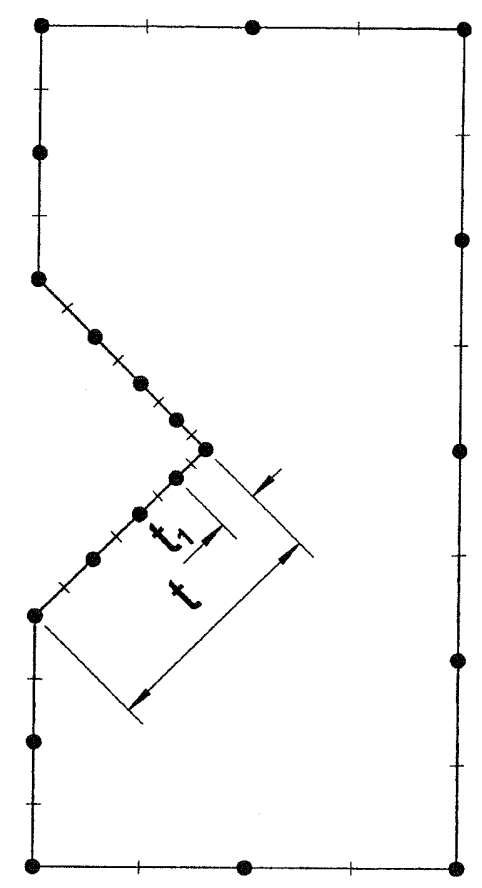

Fig. 5 Boundary element reference mesh

least-squares fitting routine available in any commercial spreadsheet (in particular, here the software GRAPHER v.4 was used) a better estimate of the parameters $Y$ is obtained at $r=0$, i.e. at the notch apex. The results obtained for the example case are shown in Table 1, where both linear extrapolation and quadratic extrapolation have been used. The results are very accurate already with mesh 2 but, from the refinement of mesh 3 and above, the results are indistinguishable from those reported in the literature. With a quadratic fitting, the results are more accurate at convergence but present a greater sensitivity with a small number of data.

\section{CONCLUSIONS}

A simple method has been presented for obtaining the NSIFs of a notched structure by processing radial stress data along the free edges of the notch in the proximity of the apex. These are likely to be the simplest data to obtain experimentally and also numerically, and therefore the

Table 1 Parameters $Y$ for the problem in Fig. 4 (reference [9] gives $Y_{\lambda_{\mathrm{s}}}=2.473$ and $Y_{\lambda_{\mathrm{al}}}=0.151$ )

\begin{tabular}{llllll}
\hline & \multicolumn{2}{c}{ Linear extrapolation } & & \multicolumn{2}{c}{ Quadratic extrapolation } \\
\cline { 2 - 3 } \cline { 5 - 6 } Mesh & $Y_{\lambda_{\mathrm{s} 1}}$ & $Y_{\lambda_{\mathrm{s} 1}}$ & & $Y_{\lambda_{\mathrm{s} 1}}$ & $Y_{\lambda_{\mathrm{a} 1}}$ \\
\hline 1 & 2.737 & 0.152 & & 4.121 & 0.152 \\
2 & 2.527 & 0.156 & & 2.979 & 0.153 \\
3 & 2.489 & 0.151 & & 2.463 & 0.150 \\
4 & 2.484 & 0.150 & & 2.472 & 0.149 \\
\hline
\end{tabular}

S07598 (C) IMechE 2000 present paper gives specific formulae to correlate these with standard definition of N-SIFs. The results with a simple numerical experiment show that it is simple to obtain quite accurate results of both mode I and mode II N-SIFs, with no need for other than standard least-squares fitting routines.

\section{ACKNOWLEDGEMENTS}

G.D. is pleased to acknowledge the support from the Consiglio Nazionale delle Ricerche (short-term Fellowship) for his visit to the University of Southampton, where the work was completed, and for the kind invitation from Professor Andy Keane, director of the Department of Mechanical Engineering.

\section{REFERENCES}

1 Williams, M. L. Surface stress singularities from various boundary conditions in angular corner of plates in extension. J. Appl. Mechanics, 1952, 74, 523-28.

2 Gregory, R. D. Green's functions, bi-linear forms, and completeness of the eigenfunctions for the elastostatic strip and wedge. J. Elasticity, 1979, 9, 283-309.

3 Tsamasphyros, G. and Theocaris, P. S. On the solution of the sector problem. J. Elasticity, 1979, 9, 271-281.

4 Demelio, G. and Pappalettere, C. Stress field near sharp notches. J. Mater. Processing Technol., 1992, 32, 155-160.

5 Rosel, R. On the wedge/notch eigenvalues. Int. J. Fracture, 1987, 33, 61-71.

6 Gross, R. and Mendelson, A. Plane elastostatic analysis of Vnotched plates. Int. J. Fracture Mechanics, 1972, 8(3), 267-276.

7 Lin, K. Y. and Tong, P. T. Singular finite element for the fracture analysis of V-notched plates. Int. J. Numer. Meth. Engng, 1980, 15, 1343-1354.

8 Szabò, A. and Babuska, I. Computation of the amplitude of stress singular terms for cracks and reentrant corners. In Proceedings of the 19th Symposium on Fracture Mechanics (Ed. T. A. Cruse), 1988, pp. 101-124 ASTM STP 969, (American Society for Testing and Materials, Philadelphia, Pennsylvania).

9 Portela, A. and Aliabadi, M. H. Efficient boundary element analysis of sharp notched plates. Int. J. Numer. Meth. Engng, 1991, 32, 445-470.

10 Atzori, B., Lazzarin, P. and Tovo, R. Stress distribution for V-shaped notches under tensile and bending loading. Fatigue Fracture Engng Mater. Structs, 1997, 20(8), 1083-1092.

11 Chen, D. H. Stress intensity factors for V-notched strip under tension or in-plane bending. Int. J. Fracture, 1995, 70, 81-97.

12 Hobbacher, A. Stress intensity factors of welded joints. Engng Fracture Mechanics, 1993, 46(2), 173-182.

13 Hobbacher, A. Stress intensity factors of welded joints. Engng Fracture Mechanics, 1994, 49(2), 323.

14 Kihara, S. and Yoshi, A. A strength evaluation method of a sharply notched structure by a new parameter, 'the equivalent stress intensity factor'. Japan Soc. Mech. Engrs Int. J., Ser. ISolid Mechanics Strength of Materials, 1991, 34(1), 70-75.

15 Nui, L. S., Chehimi, C. and Pluvinage, G. Stress field near a 
large blunted tip V-notch and application of the concept of the critical notch stress intensity factor (NSIF) to the fracture toughness of very brittle materials. Engng Fracture Mechanics, 1994, 49(3), 325-335.

16 Hobbacher, A., et al. Recommendation on fatigue of welded components. IIW document XIII-1539-95, 1995.

17 Boukharouba, T., Tamine, T., Niu, L., Chemini, C. and Pluvinage, G. The use of notch stress intensity factor as a fatigue crack initiation parameter. Engng Fracture Mechanics, 1995, 52(3), 503-512.
18 Dunn, M. L., Suwito, W. and Cunningham, S. Fracture initiation at sharp notches: correlation using critical stress intensities. Int. J. Solids Structs, 1997, 34(29), 3873-3883.

19 Dunn, M. L., Suwito, W., Cunningham, S. and May, C. W. Fracture initiation at sharp notches under mode I, mode II and mild mixed mode loading. Int. J. Fracture, 1997, 84, 367-381.

20 Barber, J. R. Elasticity, 1992 (Kluwer, Dordrecht).

21 Algorithm 365: 'complex root finding'. Collected algorithm from ACM Library. 